\title{
ANOTHER PROOF OF MONOTONICITY FOR THE EXTENDED MEAN VALUES
}

\author{
SU-LING ZHANG, CHAO-PING CHEN AND FENG QI
}

Abstract. We provide another proof of monotonicity for the extended mean values.

Stolarsky defined in [5] the extended mean values $E(r, s ; x, y)$ by

$$
\begin{array}{lll}
E(r, s ; x, y)=\left(\frac{r}{s} \cdot \frac{y^{s}-x^{s}}{y^{r}-x^{r}}\right)^{1 /(s-r)}, & & r s(r-s)(x-y) \neq 0 ; \\
E(r, 0 ; x, y)=\left(\frac{1}{r} \cdot \frac{y^{r}-x^{r}}{\ln y-\ln x}\right)^{1 / r}, & & r(x-y) \neq 0 ; \\
E(r, r ; x, y)=\frac{1}{e^{1 / r}}\left(\frac{x^{x^{r}}}{y^{y^{r}}}\right)^{1 /\left(x^{r}-y^{r}\right)}, & & r(x-y) \neq 0 ; \\
E(0,0 ; x, y)=\sqrt{x y}, & & x \neq y ; \\
E(r, s ; x, x)=x, & x=y
\end{array}
$$

and proved that it is continuous on the domain $\{(r, s ; x, y): r, s \in \mathbb{R}, x, y>0\}$.

Leach and Sholander showed in $[1,2]$ that $E(r, s ; x, y)$ is increasing with both $r$ and $s$, and with both $x$ and $y$. The monotonicities of $E$ has also been researched in [3, 4] using different ideas and simpler methods.

The aim of this article is to give another proof of monotonicity for the extended mean values $E(r, s ; x, y)$.

The variables $x$ and $y$ are, in this article, positive.

Theorem 1. E(r,s;x,y) is strictly increasing with both $r$ and $s$.

Proof. Since $E(r, s ; x, y)$ is symmetric on $r$ and $s$, it suffices to prove its monotonicity of $E(r, s ; x, y)$ with respect to $r$. Since $E(r, s ; x, y)$ is symmetric between $x$ and $y$, without loss of generality, assume $x<y$.

Received December 30, 2004.

2000 Mathematics Subject Classification. 26A48.

Key words and phrases. Monotonicity, extended mean values.

The authors were supported in part by the SF of Henan Innovation Talents at Universities, China 
Define for $r \in(-\infty,+\infty)$,

$$
\varphi(r)= \begin{cases}\ln \frac{y^{r}-x^{r}}{r}, & r \neq 0 \\ \ln \ln (y / x), & r=0\end{cases}
$$

Then,

$$
\ln E(r, s ; x, y)= \begin{cases}\frac{\varphi(r)-\varphi(s)}{r-s}, & r \neq s \\ \varphi^{\prime}(s), & r=s .\end{cases}
$$

To prove that $\ln E(r, s ; x, y)$ is strictly increasing with respect to $r$ it suffices to show that $\varphi$ is strictly convex on $(-\infty,+\infty)$. Easy computation reveals that

$$
\varphi(-r)=\varphi(r)-r \ln (x y)
$$

which implies that $\varphi^{\prime \prime}(-r)=\varphi^{\prime \prime}(r)$, and then $\varphi$ has the same convexity on both $(-\infty, 0)$ and $(0,+\infty)$. Hence, it is sufficient to prove that $\varphi$ is strictly convex on $(0,+\infty)$.

A simple computation yields

$$
r^{2} \varphi^{\prime \prime}(r)=1-\frac{(x / y)^{r}\left[\ln (x / y)^{r}\right]^{2}}{\left[1-(x / y)^{r}\right]^{2}}
$$

Define for $0<t<1$,

$$
\omega(t)=\frac{t(\ln t)^{2}}{(1-t)^{2}}
$$

Differentiation yields

$$
(1-t) t \ln t \frac{\omega^{\prime}(t)}{\omega(t)}=(1+t) \ln t+2(1-t)=-\sum_{n=2}^{\infty} \frac{n-1}{n(n+1)}(1-t)^{n+1}<0
$$

which means that $\omega^{\prime}(t)>0$ for $0<t<1$, and then, $\omega(t)<\lim _{t \rightarrow 1} \omega(t)=1$ for $0<t<1$. Clearly, $0<(x / y)^{r}<1$ for $y>x>0$ and $r>0$, and thus, $\varphi^{\prime \prime}(r)>0$ for $r>0$. The proof is complete.

\section{References}

[1] E. B. Leach and M. C. Sholander, Extended mean values, Amer. Math. Monthly 85(1978), 84-90.

[2] E. B. Leach and M. C. Sholander, Extended mean values II, J. Math. Anal. App. 92(1983), 207-223.

[3] F. Qi and Q.-M. Luo, A simple proof of monotonicity for extended mean values, J. Math. Anal. Appl. 244(1998), 356-359.

[4] F. Qi, S.-L. Xu and L. Debnath, A new proof of monotonicity for extended mean values, Internat. J. Math. Math. Sci. 22(1999), 417-421. 
[5] K. B. Stolarsky, Generalizations of the logarithmic mean, Math. Mag. 48(1975), 87-92.

Department of Basic Courses, Jiaozuo University, Jiaozuo City, Henan 454003, China.

College of Mathematics and Informatics, Henan Polytechnic University, Jiaozuo City, Henan 454010, China.

E-mail: chenchaoping@hpu.edu.cn_chenchaoping@sohu.com

Research Institute of Applied Mathematics, Henan Polytechnic University, Jiaozuo City, Henan 454010, China.

E-mail: qifeng@jzit.edu.cn fengqi618@member.ams.org 